\title{
Ability to Predict New-Onset Psychological Distress Using Routinely Collected Health Data: A Population-Based Cohort Study of Women Diagnosed With Breast Cancer
}

\author{
Ania Syrowatka, PhDa,b,c, James A. Hanley, $\mathrm{PhD}^{\mathrm{b}}$; Daniala L. Weir, $\mathrm{PhD}(\mathrm{c})^{\mathrm{a} \text {,b }}$; William G. Dixon, MRCP, PhD ${ }^{\mathrm{e}, \mathrm{f}}$; \\ Ari N. Meguerditchian, MD, MSc, FRCS ${ }^{\mathrm{a}, \mathrm{g}, \mathrm{h}}$; and Robyn Tamblyn, PhD ${ }^{\mathrm{a}, \mathrm{b}, \mathrm{i}}$
}

\begin{abstract}
Objectives: The primary objective of this study was to identify the predictors of new-onset psychological distress available in routinely collected administrative health databases for women diagnosed with breast cancer. The secondary objective was to explore whether the predictors vary based on the period of cancer care. Methods: A population-based cohort study followed 16,495 female patients with newly diagnosed breast cancer who did not experience psychological distress during the 14 months before breast cancer surgery. The incidence of psychological distress was reported overall and by type of mental health problem. Time-varying Cox proportional hazards models were developed to identify predictors of new-onset psychological distress during 2 key periods of cancer care: (1) hospital-based treatment during which women undergo treatment with breast surgery, chemotherapy, and/or radiation, and (2) 1-year transitional survivorship when women begin follow-up care. Results: The incidence of psychological distress was $16 \%$ within each period. Anxiety was present in $85.1 \%$ and $65.5 \%$ of new cases during hospital-based treatment and transitional survivorship, respectively. Predictors during both periods were younger age, receipt of axillary lymph node dissection, rheumatologic disease, and baseline menopausal symptoms, as well as new opioid dispensations, emergency department visits, and hospital contacts that occurred during follow-up. Other predictors varied based on the period of cancer care. More advanced breast cancer and type of treatment were associated with onset of psychological distress during hospital-based treatment. Psychological distress during transitional survivorship was predicted by diagnosis of localized breast disease, shorter duration of hospital-based treatment, receipt of additional hospital-based treatment in survivorship, and newly diagnosed comorbidities or symptoms. Conclusions: This study identified the predictors of new-onset psychological distress available in routinely collected administrative health databases, and showed how predictors change between hospital-based treatment and transitional survivorship periods. The results highlight the importance of developing predictive models tailored to the period of cancer care.
\end{abstract}

J Nat/ Compr Canc Netw 2018;16(9):1065-1073 doi: 10.6004/jnccn.2018.7038

$\mathbf{P}_{\text {sychological distress has been recognized as an impor- }}$ tant sequela of cancer diagnosis and treatment. ${ }^{1}$ It can have negative implications beyond affecting patients' quality of life; most importantly, psychological distress

\footnotetext{
${ }^{a}$ Clinical and Health Informatics Research Group, and bepartment of Epidemiology, Biostatistics and Occupational Health, McGill University, Montreal, Quebec, Canada; 'Canadian Foundation for Healthcare Improvement, Ottawa, Ontario, Canada; 'Institute of Health Policy, Management and Evaluation, University of Toronto, Toronto, Ontario, Canada; 'Arthritis Research UK Centre for Epidemiology, Manchester Academic Health Sciences Centre, The University of Manchester, Manchester, United Kingdom; ${ }^{~}$ Health eResearch Centre, Farr Institute, Manchester Academic Health Sciences Centre, The University of Manchester, Manchester, United Kingdom; ' ${ }^{D}$ epartment of Surgery, and hDepartment of Oncology, McGill University Health Centre, Montreal, Quebec, Canada; and 'Department of Medicine, McGill University, Montreal, Quebec, Canada.
}

has been shown to increase all-cause and cancer-related morbidity and mortality. For example, women who develop a mood disorder after a breast cancer diagnosis have an estimated $45 \%$ increased risk of all-cause mortality. ${ }^{2}$
Submitted November 1, 2017; accepted for publication April 25, 2018

The authors have disclosed that they have no financial interests, arrangements, affiliations, or commercial interests with the manufacturers of any products discussed in this article or their competitors.

Author contributions: Study concept and design: All authors. Data acquisition: Meguerditchian. Data analysis and interpretation: Syrowatka. Manuscript preparation: Syrowatka. Critical revision: Hanley, Weir, Dixon, Meguerditchian, Tamblyn.

Correspondence: Ania Syrowatka, PhD, Canadian Foundation for Healthcare Improvement, 150 Kent Street, Suite 200, Ottawa, Ontario, Canada K1P 0E4. Email: ania.syrowatka@mail.mcgill.ca 
Syrowatka et al

A possible underlying mechanism may be that psychological distress is associated with nonadherence to medical treatment in general, ${ }^{3}$ as well as cancer-related treatments, such as chemotherapy and antiestrogen therapy intended to prevent cancer recurrence. ${ }^{4,5}$

Despite these risks, diagnosis and referral of patients with cancer for appropriate psychosocial support and treatment during routine cancer care remains a challenge. Without decision-making support, oncologists have been shown to miss $61 \%$ of mild and $49 \%$ of moderate or severe cases of depression. ${ }^{6}$ Reasons range from significant overlaps in prodromal symptoms of psychological distress and treatment-related side effects (eg, insomnia and fatigue) to patients' reluctance to disclose psychological distress due to stigma around mental health problems or the assumption that psychological distress is a part of the cancer experience. ${ }^{7,8}$ Furthermore, women undergoing hospital-based treatment for breast cancer have low oncologist continuity of care, and $37 \%$ of these women attend $\leq 2$ follow-up visits with an oncologist within the first year of survivorship, limiting the number of opportunities to identify psychological distress. ${ }^{9}$ Moreover, women experiencing psychological distress may be seen less often given that they are less likely to adhere to recommended cancer care. As a result, new cases of psychological distress may not be detected.

A viable alternative to manage psychological distress could be risk stratification, which focuses on prevention rather than detection. ${ }^{10}$ This approach could help to guide allocation of limited preventive resources to manage women with an increased risk of developing psychological distress, rather than applying a uniform approach to all women regardless of risk. Risk stratification is particularly relevant in this context given that the onset of psychological distress can be prevented; specifically, prophylactic cognitive behavioral therapy has been shown to reduce the incidence of both anxiety and depression in patients with cancer at higher risk of developing psychological distress by half. ${ }^{11}$

Ideally, the risk stratification process could be automated by capitalizing on routinely collected administrative health data. These types of data are becoming increasingly available to healthcare teams, feasible to access at the point of care, and time- and resource-efficient. However, little is known about risk factors documented in administrative health da- tabases that can be used to predict onset of psychological distress. As a first step in this direction, the primary objective of this study was to identify predictors of new-onset psychological distress available in administrative health databases for women diagnosed with breast cancer. The secondary objective was to explore whether the predictors vary based on the period of cancer care, specifically during hospital-based treatment (ie, treatments received within a hospital on either an inpatient or outpatient basis, specifically breast cancer surgery, chemotherapy, and/ or radiation) and transitional survivorship (ie, first year after completion of hospital-based treatment).

\section{Methods}

\section{Study Design}

A population-based cohort of female patients with breast cancer was followed from the date of initial breast cancer surgery to 1 year after completion of hospital-based breast cancer treatment to identify cases of new-onset psychological distress (Figure 1). The cohort included women diagnosed with a new breast cancer who received hospital-based treatment in Quebec, Canada between January 1, 1998, and March 31, 2011. Breast cancer cases were identified using ICD diagnostic codes and Régie de l'assurance maladie du Québec (RAMQ) procedure codes for breast cancer surgery (see supplemental eAppendices 1 and 2, available with this article at JNCCN.org).

Women were excluded if they experienced the outcome of interest (ie, psychological distress) during the precancer baseline year or diagnostic and workup period. Women were also excluded if they did not have a documented breast surgery or were not continuously covered by the RAMQ health and drug insurance plans starting at least 14 months before the date of initial breast surgery (during the precancer baseline year and diagnostic and workup period) until completion of transitional survivorship. The date that each woman completed hospitalbased treatment was estimated based on documented RAMQ procedure codes for breast cancer surgeries, chemotherapy, and radiation, as well as consultations with medical and radiation oncologists indicating consideration of chemotherapy and radiation, respectively (supplemental eAppendix 2). Completion of hospital-based treatment was defined by the 
Prediction of Psychological Distress

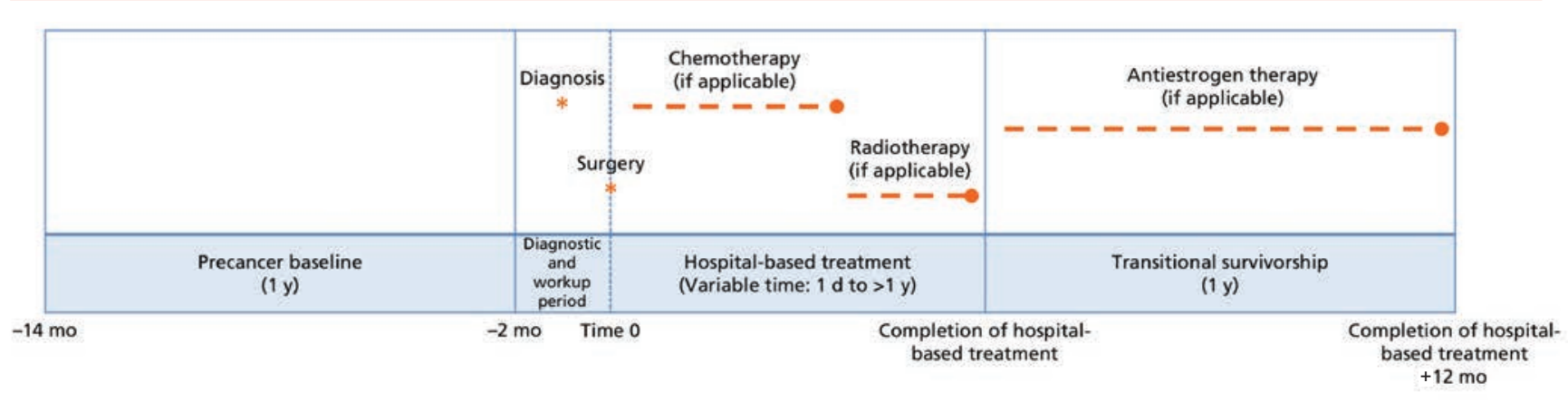

Figure 1. Periods of the cancer care trajectory: precancer baseline, hospital-based treatment, and transitional survivorship.

start of the first 6-month period without any documented hospital-based treatments for breast cancer.

\section{Data Sources}

This study was conducted using routinely collected administrative health data obtained from the RAMQ provincial, universal health insurance plan. The source population consisted of female residents from the province of Quebec, Canada insured by RAMQ. Data were available from January 1, 1998, to March 31, 2012. The following databases were linked to conduct the analyses: (1) RAMQ registrant database, which provides demographic and income-indexed prescription copayment information; (2) RAMQ medical services database, which contains physician fee-for-service claims and the ICD diagnostic code associated with each billing; (3) Ministry of Health hospital discharge abstract database (MED-ÉCHO), which provides administrative and clinical information on hospital discharges, including ICD and procedure codes; and (4) RAMQ prescription drug claims database (covering all residents aged $\geq 65$ years, and selected, dynamic subgroups aged $<65$ years). Ethical clearances were granted through the Institutional Review Board at McGill University and the provincial Access to Information Office.

\section{Measurement of Distress}

The study outcome was time to onset of new psychological distress. New episodes were identified in the administrative medical services and hospital discharge abstract databases (MED-ECHO) using documented ICD diagnostic codes for mental health problems (supplemental eAppendix 3). Given that ICD diagnostic codes for mental health problems are known to be underreported, ${ }^{12,13}$ additional new episodes of psychological distress were identified through the prescription drug claims database by dispensations of psychotropic medications commonly indicated for management of psychological distress (supplemental eAppendix 4).

\section{Candidate Predictors of Psychological Distress}

Selection of candidate predictors in the administrative health databases was informed by the published literature. All candidate predictors are listed in supplemental eAppendix 5 categorized based on type of predictor: sociodemographic information, breast cancer characteristics and treatments, comorbidities, symptoms, and health services use (ICD codes provided in supplemental eAppendices 1 and 6). When appropriate, candidate predictors were stratified by time of first documented occurrence as either "present at baseline" or "new in follow-up." This approach was taken based on evidence that side effects associated with hospital-based breast cancer treatments have been shown to increase the risk of psychological distress. ${ }^{14}$

\section{Statistical Analysis}

Descriptive statistics were calculated to characterize sociodemographic information, breast cancer characteristics, and hospital-based treatments received by the cohort. Incidence of psychological distress documented in the administrative health data was reported overall and by type: anxiety disorders, mood disorders, and other mental health problems.

Predictors of new-onset psychological distress documented in the administrative health databases were identified using time-dependent Cox proportional hazards models and the counting process approach by week of the follow-up period. Two separate models were developed: (1) hospital-based treatment and (2) transitional survivorship. Age, 
Syrowatka et al

subsidized prescription copayments (indicating lower socioeconomic status), breast cancer stage, and hospital-based treatments (for the transitional survivorship model), as well as comorbidities, symptoms, and health services use measurable prior to the start of follow-up were entered into the models as timefixed candidate predictors. Change to subsidized prescription copayments, breast cancer treatments (for the hospital-based treatment period), comorbidities, symptoms, and health services use that occurred during the follow-up period were entered into the models as time-dependent candidate predictors.

Predictors independently associated with newonset psychological distress were identified using a stepwise forward selection process with entry criteria of $P \leq .15$ for candidate predictors that were retained in the model if $P \leq .05$. The proportional hazards assumption was assessed for all predictors included in the final models by testing the significance of an interaction between each of the included predictors with linear time. Candidate interactions were entered into the final models using the same stepwise forward selection process while retaining all original predictors. All statistical analyses were conducted using SAS 9.4 (SAS Institute Inc.).

\section{Results}

The study cohort consisted of 16,495 women diagnosed with and treated for a new breast cancer who did not experience psychological distress during the precancer baseline year or diagnostic and workup period (Table 1). Incidence of psychological distress documented in the administrative health data was $16.2 \%$ (rate: 604 cases per 1,000 person-years) and $16.8 \%$ (rate: 187 cases per 1,000 person-years) during hospital-based treatment and transitional survivorship, respectively (Table 2). The most common type of psychological distress was anxiety, which was present in $85.1 \%$ of cases during hospital-based treatment and $65.5 \%$ of cases during transitional survivorship. Final models with predictors independently associated with new-onset psychological distress are presented in Table 3.

\section{Predictors Common to Both Periods of Cancer Care}

Predictors of new-onset psychological distress during both hospital-based treatment and transitional

\begin{tabular}{|c|c|}
\hline & $n(\%)$ \\
\hline \multicolumn{2}{|l|}{ Sociodemographic information at diagnosis } \\
\hline Mean age $\pm S D$ (range), y & $65.7 \pm 11.8(22-97)$ \\
\hline Subsidized prescription copayments ${ }^{\mathrm{a}}$ & $4,762(28.9 \%)$ \\
\hline \multicolumn{2}{|l|}{ Breast cancer characteristics at diagnosis } \\
\hline \multicolumn{2}{|l|}{ Stage } \\
\hline Distant & $91(0.6 \%)$ \\
\hline Regional & $3,505(21.2 \%)$ \\
\hline Localized & $10,626(64.4 \%)$ \\
\hline Uncertain & $100(0.6 \%)$ \\
\hline In situ & $2,173(13.2 \%)$ \\
\hline \multicolumn{2}{|l|}{ Hospital-based treatments received } \\
\hline \multicolumn{2}{|l|}{ Type of breast surgery } \\
\hline Mastectomy & $4,068(24.7 \%)$ \\
\hline Lumpectomy & $14,161(85.9 \%)$ \\
\hline Receipt of axillary lymph node dissection & $8,603(52.2 \%)$ \\
\hline Receipt of chemotherapy & $3,640(22.1 \%)$ \\
\hline Receipt of radiotherapy & $11,301(68.5 \%)$ \\
\hline $\begin{array}{l}\text { Mean duration of hospital-based } \\
\text { treatment, } 30 \text { days } \pm \text { SD (IQR) }\end{array}$ & $3.9 \pm 3.2(1.9-5.4)$ \\
\hline $\begin{array}{l}\text { Receipt of additional hospital-based } \\
\text { treatment in survivorship }\end{array}$ & $664(4.0 \%)$ \\
\hline
\end{tabular}

andicating lower socioeconomic status.

survivorship included younger age, receipt of axillary lymph node dissection (ALND), rheumatologic disease (present at baseline or new in follow-up), and baseline menopausal symptoms. New opioid dispensations (indicating pain), emergency department (ED) visits, and hospital contacts that occurred during the follow-up period also predicted onset of psychological distress. Breast cancer stage was independently associated with new-onset psychological distress in both periods; however, more advanced stage predicted new-onset psychological distress during hospitalbased treatment, whereas only localized breast disease predicted onset during transitional survivorship.

\section{Predictors Specific to Hospital-Based Treatment}

Additional predictors of new-onset psychological distress available in administrative health databases for the hospital-based treatment period were receipt of chemotherapy (including neoadjuvant treatment) and newly diagnosed renal disease, as well as baseline pulmonary disease, equilibrium problems, and opioid dispensations (indicating pain) that occurred 
Prediction of Psychological Distress

\begin{tabular}{|c|c|c|}
\hline Type of Psychological Distress & $\begin{array}{l}\text { Hospital-Based Treatment } \\
\qquad(\mathrm{N}=16,495)\end{array}$ & $\begin{array}{l}\text { Transitional Survivorship } \\
\qquad(\mathrm{N}=13,821)\end{array}$ \\
\hline Number of new-onset cases & $\%(n)$ & $\%(n)$ \\
\hline All psychological distress ${ }^{a}$ & $16.2 \%(2,674)$ & $16.8 \%(2,322)$ \\
\hline Anxiety & $13.8 \%(2,275)$ & $11.0 \%(1,522)$ \\
\hline Mood disorders & $2.1 \%(348)$ & $4.9 \%(681)$ \\
\hline Other mental health problems & $0.5 \%(89)$ & $1.2 \%(167)$ \\
\hline Rate of new-onset cases & Per 1,000 Person-Years & Per 1,000 Person-Years \\
\hline All psychological distress & 604 & 187 \\
\hline
\end{tabular}

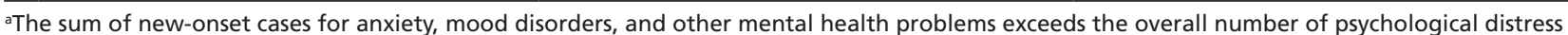
cases, because a small percentage of women with new-onset distress had multiple comorbid psychological conditions documented on the same day.

before the start of follow-up. Conversely, subsidized prescription copayments at baseline and receipt of radiotherapy were associated with a lower risk of new-onset psychological distress during hospitalbased treatment (Table 3).

\section{Predictors Specific to Transitional Survivorship}

In the transitional survivorship period, additional predictors available in the administrative health databases among women who did not experience psychological distress during hospital-based treatment included change to subsidized prescription copayments, shorter duration of hospital-based treatment, and receipt of additional hospital-based treatment in survivorship. Pulmonary symptoms (present at baseline or new in follow-up), as well as baseline gastrointestinal symptoms and pain-related diagnoses were associated with onset of psychological distress. Newly diagnosed pulmonary disease, anemia, hypothyroidism, hypertension, urinary symptoms, and fatigue also predicted new-onset psychological distress during transitional survivorship (Table 3).

\section{Discussion}

This study identified predictors of new-onset psychological distress in female patients with breast cancer documented in routinely collected administrative health databases in an effort to help guide allocation of supportive care resources. The unique contribution of this research was the identification of predictors of onset of psychological distress in administrative health databases for the early survivorship period, during which women transition to followup care and have fewer contacts with the oncology team. The documented incidence of psychological distress was $16 \%$ within each period of cancer care. Anxiety was the most common type of new psychological distress, present in $85.1 \%$ and $65.5 \%$ of new-onset cases during hospital-based treatment and transitional survivorship, respectively. Predictors of new-onset psychological distress during both periods were younger age, receipt of ALND, rheumatologic disease, and baseline menopausal symptoms, as well as new opioid dispensations, ED visits, and hospital contacts that occurred during follow-up. Predictors also varied based on the period of cancer care. More advanced breast cancer and type of treatment were associated with onset of psychological distress during hospital-based treatment. Psychological distress during transitional survivorship was predicted by diagnosis of localized breast disease, shorter duration of hospital-based treatment, receipt of additional hospital-based treatment in survivorship, and newly diagnosed comorbidities or symptoms.

Breast cancer stage at diagnosis was a significant predictor of psychological distress during both the hospital-based treatment and transitional survivorship periods; however, more advanced breast cancer was associated with new psychological distress during hospital-based treatment, whereas localized breast cancer predicted onset in the transitional survivorship period. This difference is likely attributable to the longer duration of hospital-based treatment and more aggressive anticancer therapies received by women with more advanced cancer. As a result, women with more advanced cancer who are more susceptible to psychological distress would likely develop it during hospital-based treatment, particularly while undergoing chemotherapy. Conversely, women with localized breast disease have comparatively shorter durations of hospital-based treatment, 
Syrowatka et al

\begin{tabular}{|c|c|c|}
\hline Predictor/Description ${ }^{a}$ & $\begin{array}{c}\text { Hospital-Based Treatment, } \\
\text { HR }(95 \% \mathrm{Cl}) \\
(\mathrm{N}=16,495) \\
\end{array}$ & $\begin{array}{c}\text { Transitional Survivorship, } \\
\text { HR }(95 \% \mathrm{Cl}) \\
(\mathrm{N}=13,821)\end{array}$ \\
\hline \multicolumn{3}{|l|}{ Sociodemographic information } \\
\hline Age (per 5-year increase) & $0.91(0.90-0.93)$ & $0.95(0.94-0.97)$ \\
\hline \multicolumn{3}{|c|}{ Subsidized prescription copayments (indicator of lower socioeconomic status) } \\
\hline Present at baseline: impact on day 1 & $0.79(0.69-0.91)$ & - \\
\hline Present at baseline: impact at 6 months & $1.12(0.94-1.35)$ & - \\
\hline New in follow-up & - & $1.66(1.23-2.25)$ \\
\hline \multicolumn{3}{|l|}{ Breast cancer characteristics and treatments } \\
\hline \multicolumn{3}{|l|}{ Breast cancer stage at diagnosis } \\
\hline Distant & $2.28(1.41-3.70)$ & $0.94(0.54-1.64)$ \\
\hline Regional & $1.64(1.37-1.97)$ & $1.01(0.86-1.20)$ \\
\hline Localized & $1.50(1.28-1.77)$ & $1.15(1.01-1.31)$ \\
\hline Uncertain & $1.46(0.47-4.57)$ & $0.77(0.44-1.35)$ \\
\hline In situ & Ref & Ref \\
\hline \multicolumn{3}{|l|}{ Receipt of an axillary lymph node dissection } \\
\hline Impact on day 1 & $1.25(1.11-1.41)$ & $1.52(1.31-1.75)$ \\
\hline Impact at 6 months & $0.98(0.81-1.18)$ & $1.16(1.05-1.28)$ \\
\hline \multicolumn{3}{|l|}{ Receipt of chemotherapy (including neoadjuvant treatment) } \\
\hline Impact on day 1 & $1.44(1.19-1.75)$ & - \\
\hline Impact at 6 months & $0.98(0.81-1.18)$ & - \\
\hline Receipt of radiotherapy & $0.63(0.55-0.71)$ & - \\
\hline \multicolumn{3}{|l|}{ Duration of hospital-based treatment (per additional 30 days) } \\
\hline Impact on day 1 & NA & $0.89(0.86-0.91)$ \\
\hline Impact at 6 months & NA & $0.94(0.93-0.96)$ \\
\hline Receipt of additional hospital-based treatment in survivorship & NA & $1.85(1.33-2.59)$ \\
\hline \multicolumn{3}{|l|}{ Comorbidities } \\
\hline \multicolumn{3}{|l|}{ Pulmonary disease } \\
\hline Present at baseline & $1.26(1.12-1.41)$ & - \\
\hline New in follow-up & - & $1.42(1.13-1.79)$ \\
\hline \multicolumn{3}{|l|}{ Renal disease } \\
\hline New in follow-up & $2.01(1.04-3.88)$ & - \\
\hline \multicolumn{3}{|l|}{ Rheumatologic disease } \\
\hline Present at baseline & $1.17(1.07-1.28)$ & $1.23(1.13-1.35)$ \\
\hline New in follow-up & $1.34(1.07-1.69)$ & $1.35(1.12-1.63)$ \\
\hline \multicolumn{3}{|l|}{ Anemia } \\
\hline New in follow-up & - & $1.49(1.14-1.96)$ \\
\hline \multicolumn{3}{|l|}{ Hypothyroidism } \\
\hline New in follow-up & - & $1.51(1.08-2.11)$ \\
\hline \multicolumn{3}{|l|}{ Hypertension } \\
\hline New in follow-up & - & $1.36(1.08-1.72)$ \\
\hline \multicolumn{3}{|l|}{ Equilibrium problems } \\
\hline Present at baseline & $1.28(1.03-1.58)$ & - \\
\hline
\end{tabular}

Abbreviations: HR, hazard ratio; NA, not applicable.

(continued on next page)

a8 predictors violated the proportional hazards assumption (ie, had a significant interaction with time), meaning that the HRs either increased or decreased over the follow-up period. For ease of interpretation, the HRs for these predictors are reported at 2 time points: on day 1 (ie, the beginning of follow-up) and at 6 months (ie, 6 months into the follow-up period).

in some cases limited to only breast surgery. Under these circumstances, women cope with the breast cancer diagnosis on transitioning into follow-up, where they have fewer contacts with the oncology team and reduced access to supportive care. ${ }^{9}$ These results are seemingly contrary to the conclusions of our systematic review of predictors of psychological distress in breast cancer survivors, which indicated that longer treatment duration was associated with increased risk of psychological distress. ${ }^{14}$ However, this discrepancy can be explained by the different study outcomes; the systematic review reported 
Prediction of Psychological Distress

\begin{tabular}{|c|c|c|}
\hline Predictor/Description ${ }^{\mathrm{a}}$ & $\begin{array}{c}\text { Hospital-Based Treatment, } \\
\text { HR }(95 \% \mathrm{Cl}) \\
(\mathrm{N}=16,495) \\
\end{array}$ & $\begin{array}{c}\text { Transitional Survivorship, } \\
\text { HR }(95 \% \mathrm{Cl}) \\
(\mathrm{N}=13,821)\end{array}$ \\
\hline \multicolumn{3}{|l|}{ Symptoms } \\
\hline \multicolumn{3}{|l|}{ Pulmonary symptoms } \\
\hline Present at baseline & - & $1.13(1.02-1.25)$ \\
\hline New in follow-up & - & $1.58(1.28-1.94)$ \\
\hline \multicolumn{3}{|l|}{ Urinary symptoms } \\
\hline New in follow-up: impact on day 1 & - & $4.08(1.75-9.49)$ \\
\hline New in follow-up: impact at 6 months & - & $1.89(1.27-2.79)$ \\
\hline \multicolumn{3}{|l|}{ Gastrointestinal symptoms } \\
\hline Present at baseline & - & $1.22(1.10-1.36)$ \\
\hline \multicolumn{3}{|l|}{ Menopausal symptoms } \\
\hline Present at baseline: impact on day 1 & $1.29(1.16-1.42)$ & $1.44(1.20-1.72)$ \\
\hline Present at baseline: impact at 6 months & Proportional & $1.17(1.05-1.32)$ \\
\hline \multicolumn{3}{|l|}{ Fatigue } \\
\hline New in follow-up & - & $1.83(1.33-2.52)$ \\
\hline \multicolumn{3}{|l|}{ Pain-related diagnosis } \\
\hline Present at baseline & - & $1.16(1.03-1.31)$ \\
\hline \multicolumn{3}{|l|}{ Opioid dispensation (indicator of pain) } \\
\hline Present at baseline & $1.18(1.06-1.31)$ & - \\
\hline New in follow-up: impact on day 1 & $1.08(0.90-1.29)$ & $1.24(1.01-1.51)$ \\
\hline New in follow-up: impact at 6 months & $1.56(1.29-1.87)$ & Proportional \\
\hline \multicolumn{3}{|l|}{ Health services use } \\
\hline \multicolumn{3}{|l|}{ Emergency department visit } \\
\hline New in follow-up & $1.33(1.21-1.47)$ & $1.47(1.31-1.65)$ \\
\hline \multicolumn{3}{|l|}{ Hospital contact } \\
\hline New in follow-up: impact on day 1 & $1.14(1.03-1.25)$ & $2.32(1.77-3.03)$ \\
\hline New in follow-up: impact at 6 months & Proportional & $1.70(1.48-1.96)$ \\
\hline
\end{tabular}

Abbreviations: HR, hazard ratio; NA, not applicable.

a8 predictors violated the proportional hazards assumption (ie, had a significant interaction with time), meaning that the HRs either increased or decreased over the follow-up period. For ease of interpretation, the HRs for these predictors are reported at 2 time points: on day 1 (ie, the beginning of follow-up) and at 6 months (ie, 6 months into the follow-up period).

on predictors of prevalent psychological distress, ${ }^{14}$ whereas this study investigated predictors of newonset psychological distress.

Newly diagnosed comorbidities or symptoms may have been related to hospital-based treatment. Many of the predictors could have been treatmentrelated side effects related to breast cancer surgery, chemotherapy, and/or radiation, such as pulmonary disease and related symptoms (eg, dyspnea); renal disease and urinary symptoms (eg, incontinence); anemia; hypothyroidism; hypertension; gastrointestinal symptoms (eg, nausea and vomiting); menopausal symptoms; fatigue; and pain. ${ }^{15-17}$ The results also suggest that preexisting conditions or symptoms documented during the precancer baseline and diagnostic and workup periods may have been exacerbated by receipt of hospital-based breast cancer treatments. In particular, underlying rheumatologic disease may predict onset of psychological distress as a result of discontinuation of disease-modifying immunosuppressant medications during hospital-based treatment, which would result in more pain and limited mobility. Additionally, hospital-based treatments have been shown to increase the risk of joint pain, and may serve to trigger or exacerbate underlying rheumatologic symptoms. Given the context, receipt of an ALND may predict new-onset psychological distress through increasing the risk of lymphedema, which has been shown to occur in approximately 20\% of women who receive ALNDs, based on a meta-analysis of 18 studies. ${ }^{18}$ ALND could also cause psychological distress as a result of limited joint mobility and stiffness. Results of this study highlight that treatment-related conditions and symptoms do not need to be severe in order to increase the risk of new-onset distress.

These findings also suggest that interventions focused on timely identification and effective 
Syrowatka et al

management of treatment-related symptoms could prevent onset of psychological distress by reducing the burden of disease. Electronic monitoring of treatment-related symptoms coupled with automated alerts to the oncology team has been shown to improve health-related quality of life in patients with cancer receiving chemotherapy. ${ }^{19}$ Furthermore, this intervention reduced the number of ED visits and hospitalizations. This is particularly relevant given that results of our study demonstrated that ED visits and hospital contacts either increase the risk of new psychological distress or are early signs; therefore, proactive intervention to avoid unplanned health services use may also reduce the risk of developing psychological distress.

An important strength of our study was the use of time-dependent Cox proportional hazards models that accounted for new events that occurred during the follow-up period. This approach enabled identification of newly diagnosed treatment-related comorbidities and symptoms as predictors of new-onset psychological distress, particularly during transitional survivorship. Furthermore, the time-dependent analyses helped to disentangle the independent associations of breast cancer stage and hospital-based treatments with onset of psychological distress. Previous studies have evaluated hospital-based treatments as candidate predictors of depression following breast cancer diagnosis using time-to-event models; however, the results may have been biased given that the analyses did not appear to account for the timedependent nature of these predictors. ${ }^{20,21}$ The current study also advanced the literature by stratifying the analysis by the period of cancer care, which provided more insight into the effect of duration of hospitalbased treatment on risk of new-onset psychological distress during transitional survivorship.

This study had several limitations. ICD diagnostic codes for mental health problems tend to be underreported in administrative health databases. Reasons include current RAMQ reporting structures allowing only one ICD diagnostic code to be docu- mented for physician healthcare services billing, patient reluctance to report mental health problems, and physician failure to assess psychosocial concerns during oncology visits. As a result, a substantial number of new cases of psychological distress may not have been documented. Similarly, comorbidities and symptoms may have been underreported, weakening the associations in the final models; for example, despite being relatively common after ALND, ${ }^{18}$ lymphedema was rarely documented in the administrative health databases. Conversely, measuring psychological distress based on psychotropic medication dispensations from administrative prescription drug claims databases may have overestimated the number of cases of new-onset psychological distress, also resulting in weaker associations. Some of the psychotropic medications may have been prescribed for other indications, such as sleep disorders, migraines, or pain. ${ }^{22}$ Furthermore, our study did not capture new cases of psychological distress if psychotropic medications were prescribed but never dispensed (ie, primary nonadherence $)^{23}$ or if women received alternative treatments for psychological distress not covered by the universal health insurance plan, specifically psychotherapy.

\section{Conclusions}

This study identified predictors of new-onset psychological distress available in routinely collected administrative health databases, and showed how the predictors change based on the period of cancer care, specifically during hospital-based treatment and transitional survivorship. These results indicate that a one-size-fits-all approach is not sufficient to predict new-onset psychological distress in patients with breast cancer and that predictive models should be tailored based on the period of cancer care. These findings could be used to inform development of risk stratification algorithms to identify women at higher risk of new-onset psychological distress following breast cancer diagnosis.

\section{References}

1. Adler NE, Page AE, eds. Cancer Care for the Whole Patient: Meeting Psychosocial Health Needs. Washington, DC: The National Academies Press; 2008.

2. Kanani R, Davies EA, Hanchett N, Jack RH. The association of mood disorders with breast cancer survival: an investigation of linked cancer

registration and hospital admission data for South East England Psychooncology 2016;25:19-27.

3. DiMatteo MR, Lepper HS, Croghan TW. Depression is a risk factor for noncompliance with medical treatment: meta-analysis of the effects of anxiety and depression on patient adherence. Arch Intern Med 2000;160:2101-2107. 
Prediction of Psychological Distress

4. DiMatteo MR, Haskard-Zolnierek KB. Impact of depression on treatment adherence and survival from cancer. In: Kissane DW, Maj M, Sartorius N, eds. Depression and Cancer. Chichester, United Kingdom: WileyBlackwell; 2011:101-124.

5. Mausbach BT, Schwab RB, Irwin SA. Depression as a predictor of adherence to adjuvant endocrine therapy (AET) in women with breast cancer: a systematic review and meta-analysis. Breast Cancer Res Treat 2015;152:239-246.

6. Passik SD, Dugan W, McDonald MV, et al. Oncologists' recognition of depression in their patients with cancer. J Clin Oncol 1998;16:1594-1600.

7. Smith HR. Depression in cancer patients: pathogenesis, implications and treatment. Oncol Lett 2015;9:1509-1514.

8. Corrigan PW, Druss BG, Perlick DA. The impact of mental illness stigma on seeking and participating in mental health care. Psychol Sci Public Interest 2014;15:37-70.

9. Grunfeld E, Hodgson DC, Del Giudice ME, Moineddin R. Populationbased longitudinal study of follow-up care for breast cancer survivors. J Oncol Pract 2010;6:174-181.

10. Shashank A. The importance of risk stratification in population health management. Available at: http://blog.innovaccer.com/importance-riskstratification-population-health-management. Accessed August 2, 2018

11. Pitceathly C, Maguire P, Fletcher I, et al. Can a brief psychological intervention prevent anxiety or depressive disorders in cancer patients? A randomised controlled trial. Ann Oncol 2009;20:928-934.

12. Rost K, Smith R, Matthews DB, Guise B. The deliberate misdiagnosis of major depression in primary care. Arch Fam Med 1994;3:333-337.

13. Ford E, Campion A, Chamles DA, et al. 'You don't immediately stick a label on them': a qualitative study of influences on general practitioners' recording of anxiety disorders. BMJ Open 2016;6:e010746.

14. Syrowatka A, Motulsky A, Kurteva S, et al. Predictors of distress in female breast cancer survivors: a systematic review. Breast Cancer Res Treat 2017;165:229-245.
15. Aaronson NK, Ahmedzai S, Bergman B, et al. The European Organization for Research and Treatment of Cancer QLQ-C30: a quality-of-life instrument for use in international clinical trials in oncology. J Natl Cancer Inst 2005;85:365-376.

16. Breastcancer.org. Treatment Side Effects. Available at: http://www. breastcancer.org/treatment/side_effects. Modified February 18, 2017. Accessed August 2, 2018.

17. Hurria A, Come SE, Pierce LJ. Patterns of relapse and long-term complications of therapy in breast cancer survivors. UpToDate Web site. Available at: https://www.uptodate.com/contents/patterns-of-relapse-andlong-term-complications-of-therapy-in-breast-cancer-survivors. Updated May 30, 2018. Accessed August 2, 2018.

18. DiSipio $T$, Rye $S$, Newman $B$, Hayes $S$. Incidence of unilateral arm lymphoedema after breast cancer: a systematic review and meta-analysis. Lancet Oncol 2013;14:500-515.

19. Basch E, Deal AM, Kris MG, et al. Symptom monitoring with patientreported outcomes during routine cancer treatment: a randomized controlled trial. J Clin Oncol 2016;34:557-565.

20. Suppli NP, Deltour I, Damkjaer LH, et al. Factors associated with the prescription of antidepressive medication to breast cancer patients. Acta Oncol 2011;50:243-251

21. Suppli NP, Johansen C, Christensen J, et al. Increased risk for depression after breast cancer: a nationwide population-based cohort study of associated factors in Denmark, 1998-2011. J Clin Oncol 2014;32:38313839 .

22. Wong J, Motulsky A, Eguale $\mathrm{T}$, et al. Treatment indications for antidepressants prescribed in primary care in Quebec, Canada, 2006-2015. JAMA 2016;315:2230-2232.

23. Tamblyn R, Eguale $T$, Huang A, et al. The incidence and determinants of primary nonadherence with prescribed medication in primary care: a cohort study. Ann Intern Med 2014;160:441-450.

\section{See JNCCN.org for supplemental online content.}

